\title{
Kinetic Chromogenic Assay
}

National Cancer Institute

\section{Source}

National Cancer Institute. Kinetic Chromogenic Assay. NCI Thesaurus. Code C142339.

A bioassay in which a biological specimen is incubated with a kinetic substrate, followed by optical analysis of color change. 